\title{
\begin{tabular}{|l|l|l|l|l}
\hline M & R & S & Internet Journal of & Nitride Semiconductor Research \\
\hline
\end{tabular}
}

\author{
Volume 2, Article 7
}

\section{Nonuniform Morphology and Luminescence Properties of a Molecular Beam Epitaxy GaN Film from Atomic Force Microscopy, Scanning Electron Microscopy and Cathodoluminescence}

\author{
L.-L. Chao, G. S. Cargill III, C. Kothandaraman \\ Department of Chemical Engineering, Materials Science, and Mining Engineering, Columbia University \\ D. Cyr, G. Flynn \\ Department of Chemistry, Columbia University \\ E. S. Hellman, D. Wiesmann, D. N. E. Buchanan, I. Brener \\ Bell Laboratories, Lucent Technologies
}

This article was received on December 30, 1996 and accepted on May 23, 1997.

\begin{abstract}
Complex faceted features of micrometer sizes and with intense luminescence rise 200-300 nm above the surface of a GaN thin film grown by molecular beam epitaxy on (0001) sapphire. Cathodoluminescence measurements at room temperature and at $8 \mathrm{~K}$ were used to investigate the luminescence properties of these microfeatures in comparison with those of the background GaN material. The morphology of the micro-features was studied by scanning electron microscopy and by atomic force microscopy.

$\mathrm{GaN}$ and related compounds have been considered as promising materials for light emitting devices in the short wavelength visible and UV spectral regions because of their direct, wide band gaps and high luminescence efficiency. Remarkable, rapid success has been achieved in developing devices based on III-V nitrides, which allows these material systems to rival other systems under development[1]. Blue-green LEDs fabricated from InGaN/AIGaN double-heterostructure layers on sapphire substrates by Nichia Chemical Industries in Japan are commercially available, despite the presence of high extended defect densities in the $10^{10} / \mathrm{cm}^{2}$ range [2]. In December 1995, an important milestone in the development of nitride lasers was reached; Nakamura et al. in Nichia demonstrated the first nitride-based laser diode which operated at $417 \mathrm{~nm}$ under pulsed conditions at room
\end{abstract} temperature [3].

The morphology and spatial distribution of luminescence in GaN thin films have been investigated by several research groups [4], [5], [6], although most efforts have been directed to developing appropriate growth conditions for improved film quality. From observations by scanning electron microscopy (SEM)and atomic force microscopy (AFM), Trager-Cowan et al. described an MBE-grown GaN film which contained an assembly of oriented hexagonal crystallites rising above a background of polycrystalline or amorphous material [4]. All the crystallites, oriented in a similar fashion, had roughly the same sizes, 1 or $2 \mu \mathrm{m}$ across a hexagonal face and about $1 \mu \mathrm{m}$ high. These crystallites were much brighter than the surrounding background material in panchromatic low-temperature cathodoluminescence $(\mathrm{CL})$ images. Trager-Cowan et al. concluded that the crystallites were of better quality than the background material. They also observed a green emission band, attributed to impurities, which became weaker for higher electron beam voltages, generating luminescence from deeper in the film. From this observation, they concluded that higher quality material is located closer to the film's outer surface. Spatial variation of the luminescence efficiency from MOCVD-grown films has also been observed by Ponce et al[5]. in their room-temperature CL microscopy studies. Their results showed significant nonuniformities in both the band-edge and yellow band emissions. Although they reported no faceted island structures, one of their samples had "marked surface features" and consisted of hexagonal crystals 10 to $50 \mu \mathrm{m}$ in diameter. The crystals gave strong band-to-band (364 $\mathrm{nm}$ ) luminescence.

In this paper, the morphology of a GaN film grown on (0001) sapphire by MBE is characterized by SEM and AFM, and CL measurements are used to investigate the luminescence properties of the film at room temperature and at 
8K. Some regions of this film are similar to the one described by Trager-Cowan et al. [4] in having micron sized, brightly luminescent islands. The hexagonal facet angles and bright luminescence of islands in our film also resemble the larger scale, more regularly shaped hexagonal crystals of Ponce et al. [5]. These regions lie on the boundary of excess gallium growth conditions. Our results differ from those of Trager-Cowan in terms of the island morphologies and the spectral character of luminescence from the islands and from the background material.

Possible causes of the large variations in luminescence efficiency are discussed.

\section{Experimental}

\subsection{Sample Preparation}

Nitride growth was done in a Riber molecular beam epitaxy system with a custom nitrogen plasma source. The plasma source is described in Ref. [7]. The nitrogen plasma is excited with 10-15 watts of RF power in nitrogen at 65-85 mTorr. The typical plume of the plasma source is only $2 \mathrm{~cm}$ wide at the $7.5 \mathrm{~cm}$ diameter substrate holder, resulting in spatially varying active nitrogen flux. The films described here were doped with magnesium at relatively high levels. The (0001) sapphire substrate was heated to $675^{\circ} \mathrm{C}$ in vacuum, then exposed to the nitrogen plasma at a temperature of $600^{\circ} \mathrm{C}$ for 30 minutes before starting the GaN deposition. The reflection high energy electron diffraction (RHEED) pattern was observed through the whole process. The first 30 minutes of growth were done with the Ga effusion cell adjusted to obtain a slow growth rate of about $50 \mathrm{~nm} / \mathrm{hr}$, a substrate temperature of $600^{\circ} \mathrm{C}$, and a plasma power of 10W. This initial growth produced a streaky RHEED pattern indicative of a substantially smooth, crystalline surface. The growth was then continued with the Ga cell set to grow $150 \mathrm{~nm} / \mathrm{hr}$; the substrate temperature was raised to $650^{\circ} \mathrm{C}$; and the plasma power was increased to $15 \mathrm{~W}$. At the end of the growth, the film had a very streaky RHEED pattern. The film thickness was $0.49 \mu \mathrm{m}$ thick, as determined by the interference fringes in optical transmission spectroscopy.

\subsection{Atomic Force Microscopy}

The GaN film was imaged with a Nanoscope III (Digital Instruments) contact mode AFM under ambient conditions. A $200 \mu \mathrm{m}$, triangular, silicon nitride cantilever ( $\mathrm{k}=0.12 \mathrm{~N} / \mathrm{m}$, Digital Instruments) was selected to perform the imaging. The sample was secured on a steel disk with double sided tape before being placed on a magnetic, $12 \mu \mathrm{m}$ scanner. In a random $12 \mu \mathrm{m}$ scan of the surface only one $\mathrm{GaN}$ island or a portion of a $\mathrm{GaN}$ island could be observed. However, the x-y translation stage of the cantilever mount allowed several different GaN islands to be "centered" within the range of the scanner. The images were stored as $512 \times 512$ point arrays and no image processing was necessary.

\subsection{Cathodoluminescence}

The instrument used for CL measurements is based on a JEOL JSM-6400 scanning electron microscope equipped with an Oxford Instrument CF302 system, which allows measurements to be made between room temperature and $8 \mathrm{~K}$. The CL system is described in a previous publication [8]. CL measurements were made at room temperature and at $8 \mathrm{~K}$ with $15 \mathrm{kV}$ beam voltage and $60 \mathrm{nA}$ beam current.

\subsection{Photoluminescence}

Photoluminescence $(\mathrm{PL})$ measurements of the films were made at $5 \mathrm{~K}$ and at room temperature. A He-Cd laser was used as an excitation source; the emitted light was dispersed by a $0.5 \mathrm{~m}$ monochromator and detected by a CCD camera. PL spectra were measured at 7 spots along the full sample to determine the spatial uniformity of the film.

\subsection{X-ray Diffraction}

X-ray diffraction measurements of the films were made using a 4-circle diffractometer with monochromatized Cu K $\alpha$ radiation.

\section{Results and Discussions}

Inspection of the film by optical microscopy (Figure 1a) revealed interesting spatial variation of the film morphology. A circular region about $2 \mathrm{~cm}$ in diameter was essentially smooth and featureless. Outside this region, the smooth film was punctuated with gallium droplets, about 2-3 $\mu \mathrm{m}$ in diameter, spaced roughly 5-30 $\mu \mathrm{m}$ apart. In an annulus about $1 \mathrm{~mm}$ wide separating the regions with and without droplets was a region which looked much like the dropleted region, except that instead of droplets there were features with a texture too fine to resolve in the optical 
microscope.

The SEM micrograph in Figure $1 \mathrm{~b}$ shows these densely-distributed features on the surface of the as-grown film. These island-like features are $2-3 \mu \mathrm{m}$ in diameter, and separations between islands vary from $5 \mu \mathrm{m}$ to $30 \mu \mathrm{m}$. The typical morphology of these features, shown in Figure 1c, consists of entangled submicron faceted structures with many hexagonal $\left(120^{\circ}\right)$ facet angles. Observations by AFM, as shown in Figure 1d, give similar results for the feature morphologies, although the micrographs shown in Figure 1c and Figure 1d are from different islands. Figure 1e shows the three-dimensional AFM image recorded from the island used in Figure 1d, which shows that the island is rising above the surface by about $250 \mathrm{~nm}$. As described earlier in this paper, an inhomogeneous surface morphology for GaN films had been reported by Trager-Cowan et al. [4]. However, the features on the surface of our sample are labyrinthine faceted structures, unlike the simple hexagonal plates or hillocks reported in Ref. [4], or the raised hexagonal crystals in Ref. [5]. The background material for our sample is highly epitaxial, both from RHEED patterns observed during growth, as shown in Figure 2a, and from the electron channeling pattern [9], as shown in Figure $2 \mathrm{~b}$, obtained from an area including both islands and background material. This was further confirmed by the $x$-ray diffraction measurements, which showed only the GaN (0001) orientation grown on the (0001) sapphire. No other diffraction planes were observed close to the substrate normal, even in scans with wide-open slits.

Spatially-resolved CL measurements were made to relate the luminescence efficiency to the microstructures described above. As shown in Figure 3, only a broad, asymmetric peak was observed in the CL spectra obtained at room temperature from (a) one of the islands and (b) its neighboring background material. The peak wavelengths, $364.8 \mathrm{~nm}$ and 367.0 for luminescence from island and from background material respectively, are attributed to band-edge emission [5] [10]. The band-edge luminescence from the island has a narrower spectrum than that of the neighboring background material, and the luminescence intensity of the island is an order of magnitude higher, although both spectra shown in Figure 3 have been scaled to have the same peak height. The appearance of noise spikes in the spectrum obtained from background material is due to the poor signal-to-noise ratio.

The ratio of luminescence intensity between an island and its neighborhood could be as high as two orders of magnitudes, as shown in Figure 4 from measurements on five islands and their corresponding neighborhoods. Figure 4 also shows that the luminescence efficiency varies from island to island, while it is spatially uniform in the background material. The monochromatic CL image, shown in Figure 5, recorded at the peak luminescence energy from the same area used for the SEM image in Figure 1b, also confirms the luminescence contrast between islands and background material.

In addition to spatial variation of luminescence intensity, a spectral shift of 1-2 $\mathrm{nm}$ in peak wavelength was observed between individual islands and the neighboring background material, as shown in Figure 6. The peak luminescence of each island always had a shorter wavelength than that of the corresponding neighborhood. It is well known that high impurity concentration can lead to tails of states extending as impurity bands into the forbidden energy gap, and that this will cause a smaller energy gap and a broader luminescence [a]. Therefore, the blue shift and narrowing of the luminescence from the islands might result from the islands having lower $\mathrm{Mg}$ doping and fewer impurity- or defect-related near band-edge, or band-tail states than the background material. Luminescence from the islands would then be mainly band-to-band, and luminescence >from the background material would be red shifted because it mainly involved band-tail states. We speculate that the islands may lower $\mathrm{Mg}$ concentration than the background material because they are formed by liquid phase epitaxy from Gadroplets at the edge of the nitrogen plasma beam, as discussed below. The lower incident nitrogen flux responsible for forming the islands is expected to give a lower flux of oxygen from the ppm level water impurity in the nitrogen source. Less oxygen in the islands is expected to reduce incorporation of $\mathrm{Mg}$ as a co-dopant [12] relative to $\mathrm{Mg}$ incorporation in the background material.

$\mathrm{CL}$ spectra recorded at $8 \mathrm{~K}$ from one of the islands and from its neighboring background material are shown inFigure 7 (a) and (b). Three main features appear in the spectrum from the island. (1) An intense peak at $357.2 \mathrm{~nm}$ with a FWHM of $5.2 \mathrm{~nm}$ is associated with excitons bound to neutral acceptors (BE), which has been previously reported [13], [14]. (2) A weaker, broader peak attributed to recombination at donor-acceptor (DA) pairs occurs at $375.4 \mathrm{~nm}$ [15]. (3) An LO-phonon replica of the DA transition occurs at $385 \mathrm{~nm}$. The BE emission at low temperature from background material was also found to be red shifted by less than $1 \mathrm{~nm}$ with respect to that from islands in five different measurements. In these measurements, as the electron beam was moved from an island to its neighboring material, the intensity of bound exciton emission was reduced by factors of 80-100, while the relative intensity of bound exciton emission to donor-acceptor-pair transition dropped by a factor of about two. In addition to the spectral features described above, a broad, weak emission band, centered at $\sim 490 \mathrm{mn}$, appeared in the spectra of the background material, which is probably associated with the deep levels from impurities or structure defects. However, our observed impurity-induced luminescence band is more greenish than in other reported data, which place the impurity luminescence band at $550 \mathrm{~nm}$ [16], [17]. Our observations suggest that the GaN material in the islands has fewer impurities and/or structure defects than the background material, in agreement with the suggestions by Trager-Cowan et al [4]. 
PL measurements were consistent with the CL measurements. Strong, spatially inhomogeneous band-edge luminescence was observed at $300 \mathrm{~K}$ and at $5 \mathrm{~K}$ in the annular region where the faceted islands were seen, whereas only weak band-edge luminescence was observed in the smooth, featureless center region. At $5 \mathrm{~K}$ the difference is particularly striking as shown in Figure 8. PL from the smooth center region has almost no band-edge peak, with most of the luminescence coming in the broad band between $380 \mathrm{~nm}$ and $390 \mathrm{~nm}$. This band is probably associated with $\mathrm{Mg}$ acceptors [13], [18]. The difference between the CL spectrum of Figure $7 \mathrm{~b}$ and the PL spectrum of Figure $8 \mathrm{~b}$ may be due to the higher excitation density for $\mathrm{CL}$, or to intrinsic differences between the background material from the halo region and the smooth, featureless center region.

The cause of the spatial variation of the film morphology is no mystery; Ga droplets form in the region outside the direct plasma beam as a result of insufficient active nitrogen. GaN is thermodynamically unstable under conditions used in MBE, and it only forms because the decomposition to $\mathrm{Ga}$ and $\mathrm{N}_{2}$ is slow compared to the formation of GaN in active nitrogen [19]. In the plasma beam, Ga droplet formation is suppressed, and atomically smooth (0001) facets grow by step flow or layer-by-layer growth typical of MBE. It is the region at the boundary which is a puzzle: what are the faceted islands, why and how do they form, and why is the luminescence so much brighter?

The size and distribution of the faceted islands are identical to those of the droplets in the outer region of the film; this suggests that the two morphologies have a common origin. One possibility is that Ga droplets are formed in regions out of the plasma beam during the low temperature nucleation step. Droplets near the edge of the plasma beam are then consumed, forming high quality GaN from a nitrogen saturated melt in a process similar to that demonstrated by Argoitia et al. [20]. Another possibility is that GaN islands of a different polarity or orientation form in the initial growth step, and then nucleate droplets outside of the beam and high quality GaN at the edge. However, we found no evidence for this in the $x$-ray diffraction.

\section{Conclusions}

The morphology of an MBE-grown GaN film on a (0001) sapphire substrate was characterized by SEM and AFM. Multiple-faceted features of 2-3 $\mu \mathrm{m}$ in diameter rose above the film surface by about $250 \mathrm{~nm}$. Differences in luminescence intensity and spectral shifts were observed in CL spectra recorded at both room temperature and $8 \mathrm{~K}$ from island features and from the background material. The luminescence intensity of any individual island was always two orders of magnitude or more greater than that of neighboring background material.

The high luminescence from the faceted islands is a tantalizing mystery. It might be a result of better crystallinity, or reduced impurities. Determining the cause is the next topic for investigation and could lead to improved growth techniques.

\section{Footnotes}

[a]Ref. [11], Page 132

\section{References}

[1] T. Matsuoka, A. Ohki, T. Ohno, Y. Kawaguchi, J. Cryst. Growth 138, 727-736 (1994).

[2] S. D. Lester , F. A. Ponce, M. G. Craford, D. A. Steigerwald , Appl. Phys. Lett. 66, 1249-1251 (1995).

[3] S Nakamura, M Senoh, S Nagahama, N Iwasa, T Yamada, T Matsushita, H Kiyoku, Y Sugimoto, Jpn. J. Appl. Phys. 35, L74-L76 (1996).

[4] C. Trager-Cowan, K. P. O'Donnell , S. E. Hooper, C. T. Foxon , Appl. Phys. Lett. 68, 355-357 (1996).

[5] F. A. Ponce, D. P. Bour, W. Gotz , P. J. Wright , Appl. Phys. Lett. 68, 57-59 (1996).

[6] B. Garni, Jian Ma, N. Perkins, Jutong Liu, T. F. Kuech, M. G. Lagally , Appl. Phys. Lett. 68, 1380-1382 (1996).

[7] R. J. Spah, H. F. Hess, H. L. Stormer, A. E. White, K. T. Short, Appl. Phys. Lett. 53, 441-443 (1988).

[8] L. -L. Chao, M. B. Freiler, M. Levy, J. -L. Lin, G. S. Cargill III, R. M. Osgood Jr, G. F. McLane,Mater. Res. Soc. Symp. Proc. 406, 543-548 (1996). 
[9] D. C. Joy, D. E. Newbury, D. L. Davidson, J. Appl. Phys. 53, R81-R122 (1982).

[10] W. Shan, X. C. Xie, J. J. Song , B. Goldenberg , Appl. Phys. Lett. 67, 2512-2514 (1995).

[11] J. I. Pankove, Optical Processes in Semiconductors (Dover Publications, New York, 1971) .

[12] T. Yamamoto and H. Katayama-Yoshida, "Control of valence states by a codoping method in p-type GaN materials,» paper D4.2, Spring 1997 MRS Meeting, San Francisco

[13] C. Merz, M. Kunzer, U. Kaufmann, Semicond. Sci. Technol. 11, 712 (1996).

[14] S Fischer, C Wetzel, EE Haller, BK Meyer, Appl. Phys. Lett. 67, 1298-1300 (1995).

[15] W Rieger, T Metzger, H Angerer, R Dimitrov, O Ambacher, M Stutzmann, Appl. Phys. Lett. 68,970 (1996).

[16] E. R. Glaser, T. A. Kennedy, K. Doverspike, L. B. Rowland, D. K. Gaskill , J. A. Freitas, Jr., M. Asif Khan, D. T. Olson, J. N. Kuznia, D. K. Wickenden, Phys. Rev. B 51, 13326-13336 (1995).

[17] J. A. Freitas, T. A. Kennedy, E. R. Glaser, W. E. Carlos, Sol. St. Electr. 41, 185-188 (1997).

[18] JM Myoung, KH Shim, C Kim, O Gluschenkov, K Kim, S Kim, DA Turnbull, SG Bishop, Appl. Phys. Lett. 69, 2722-2724 (1996).

[19] N. Newman, J. Ross, M. Rubin , Appl. Phys. Lett. 62, 1242-1244 (1993).

[20] A. Argoitia, C. C. Hayman, J. C. Angus, L. Wang, J. S. Dyck, K. Kash, Appl. Phys. Lett. 70, 179-181 (1997).

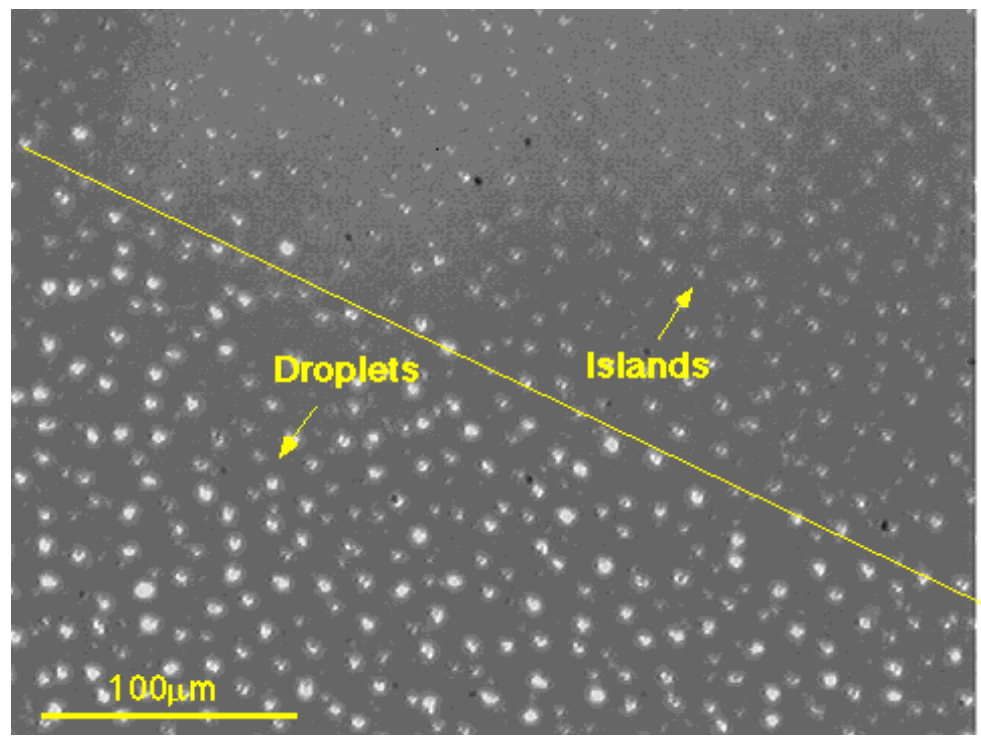

Figure 1a. Optical micrograph of a GaN film showing the boundary region, indicated by the dashed line, between Ga droplets (bottom, left) and GaN islands (top, right). The field of view is $400 \mu \mathrm{m} \times 280 \mu \mathrm{m}$. 


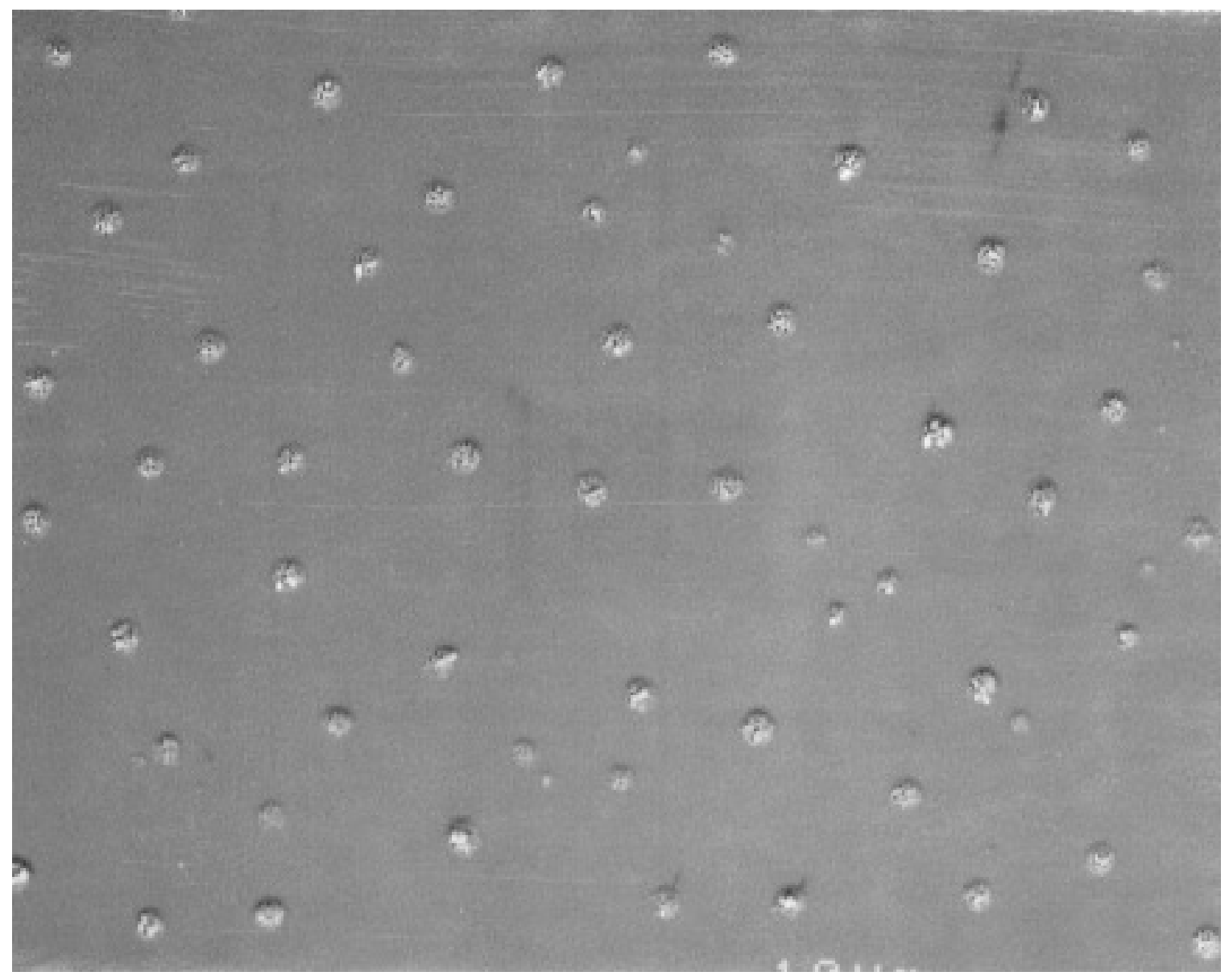

Figure 1b. Top view SEM image showing islands of micron size on the surface of the GaN film. 


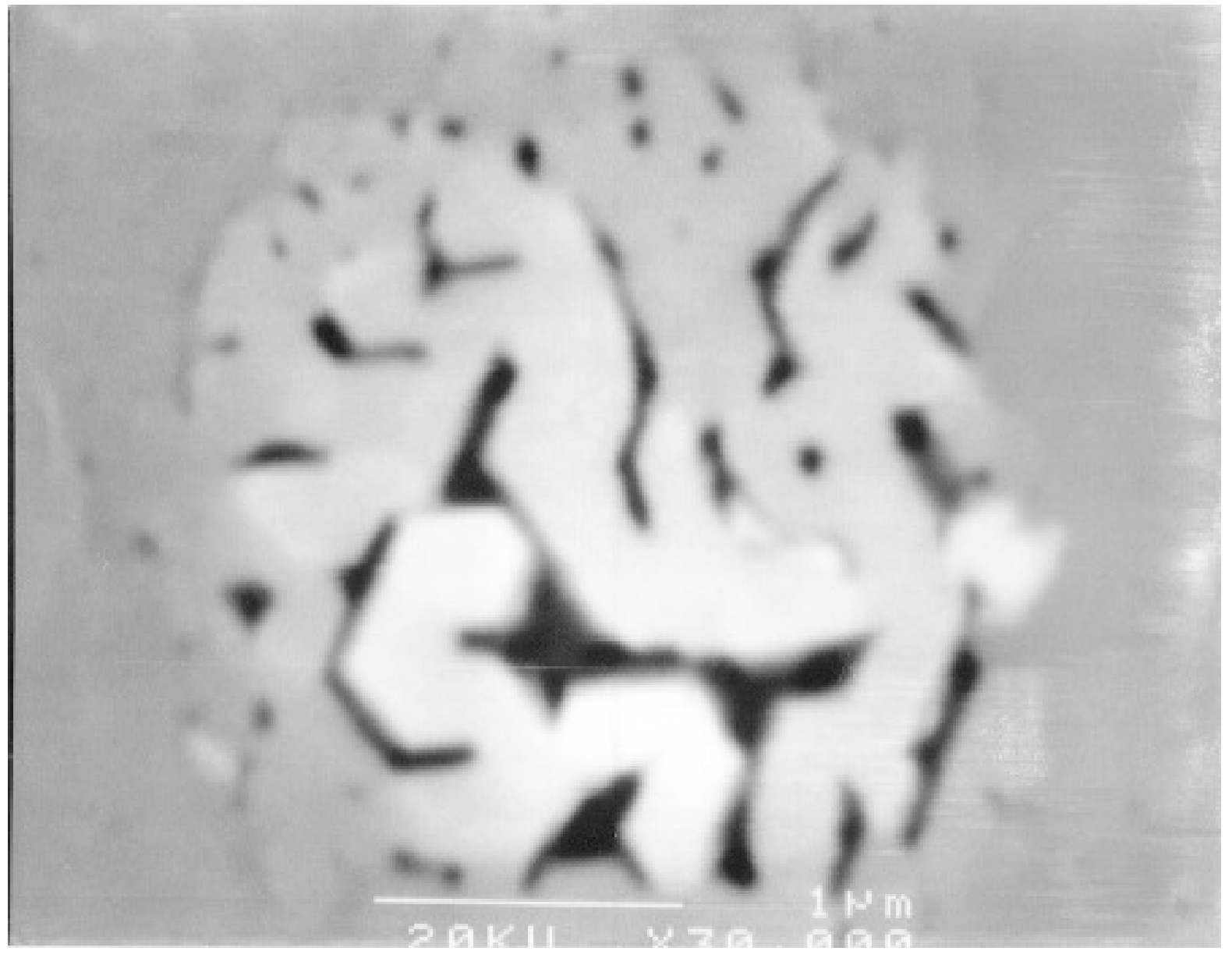

Figure 1c. Top view SEM image of one of the islands. 


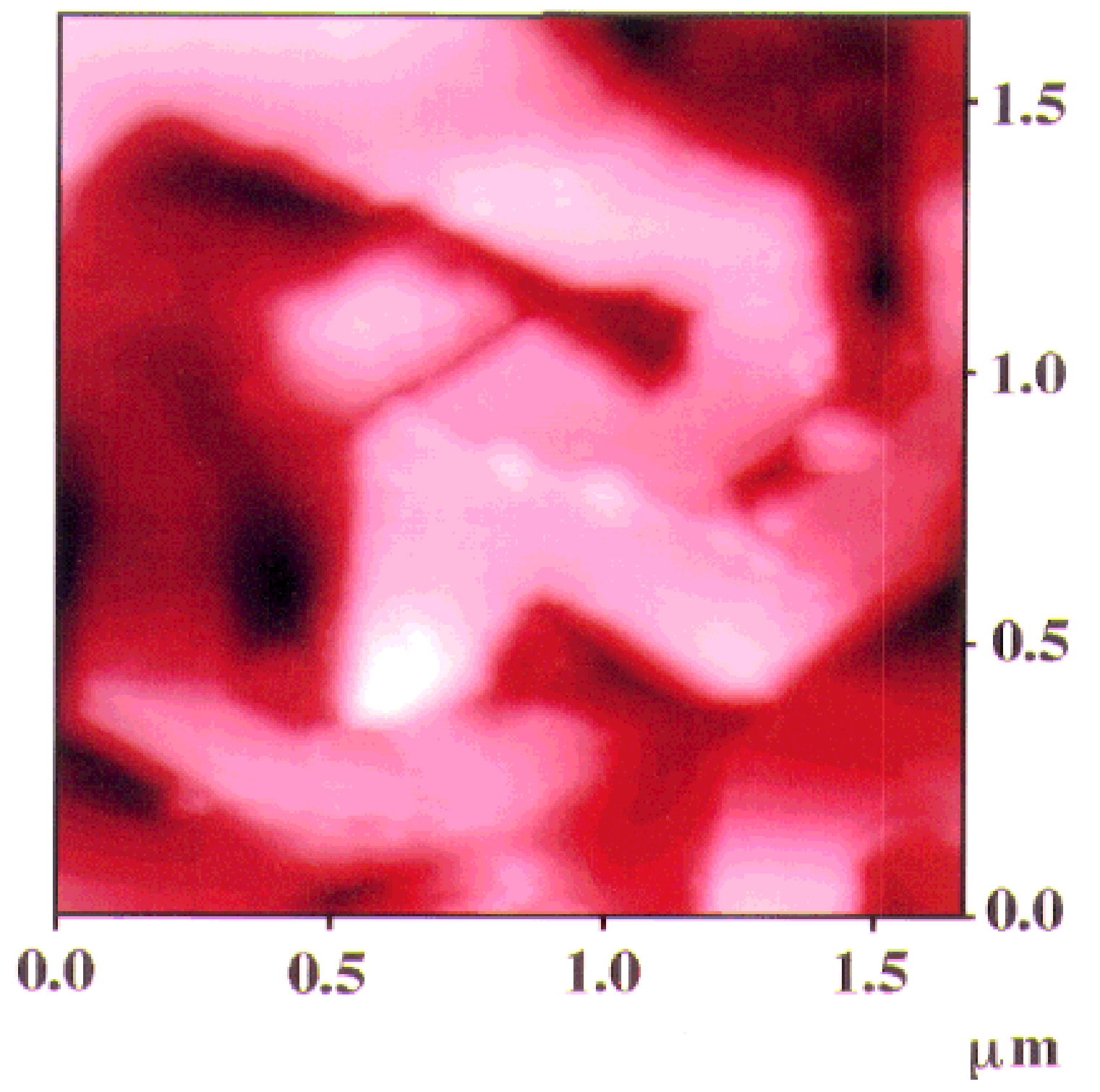

Figure 1d. Top view AFM image of one of the islands. 


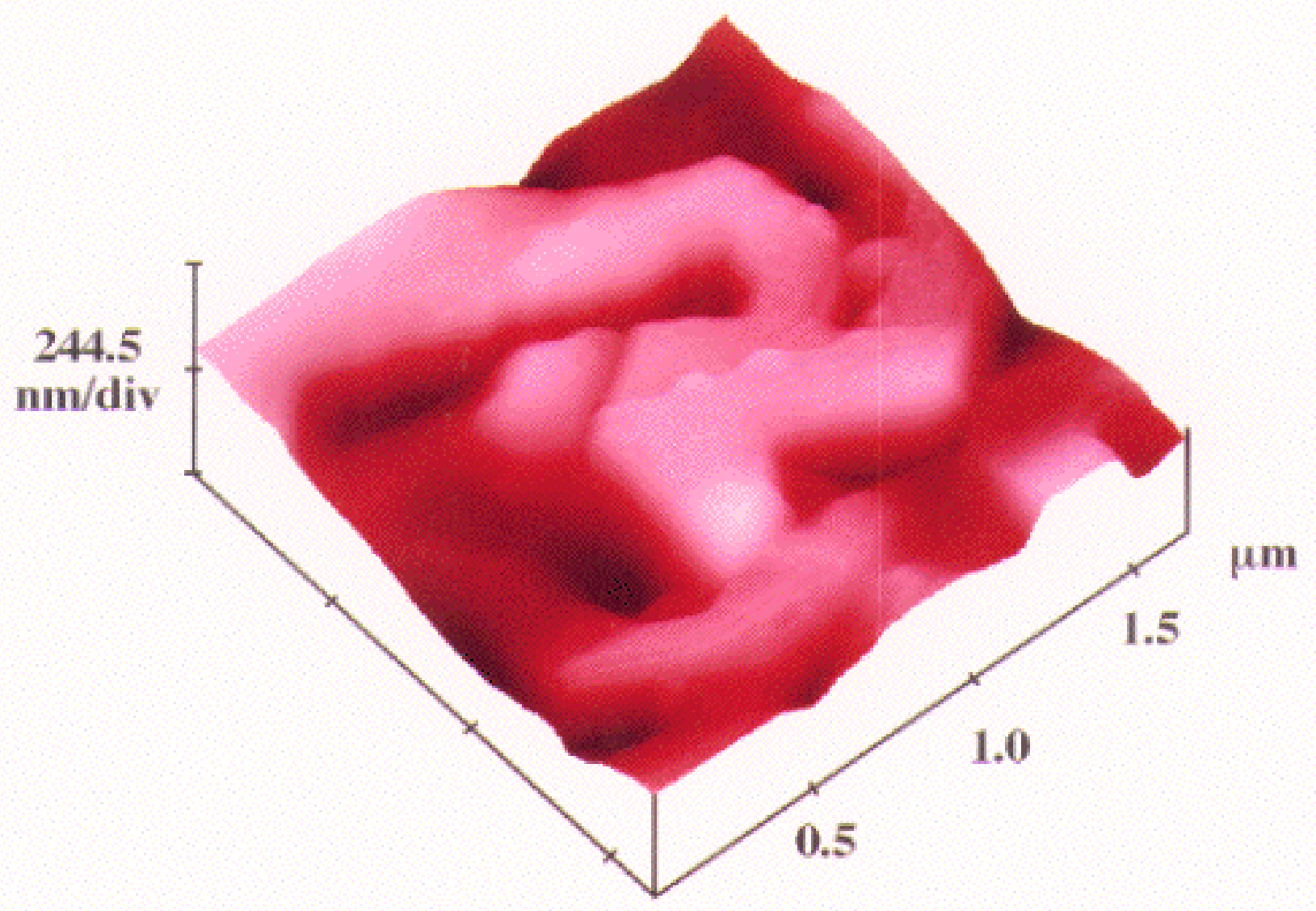

Figure 1e. Perspective view AFM image showing the height of the island. 
Figure 2a. RHEED pattern observed during the growth of the film. 


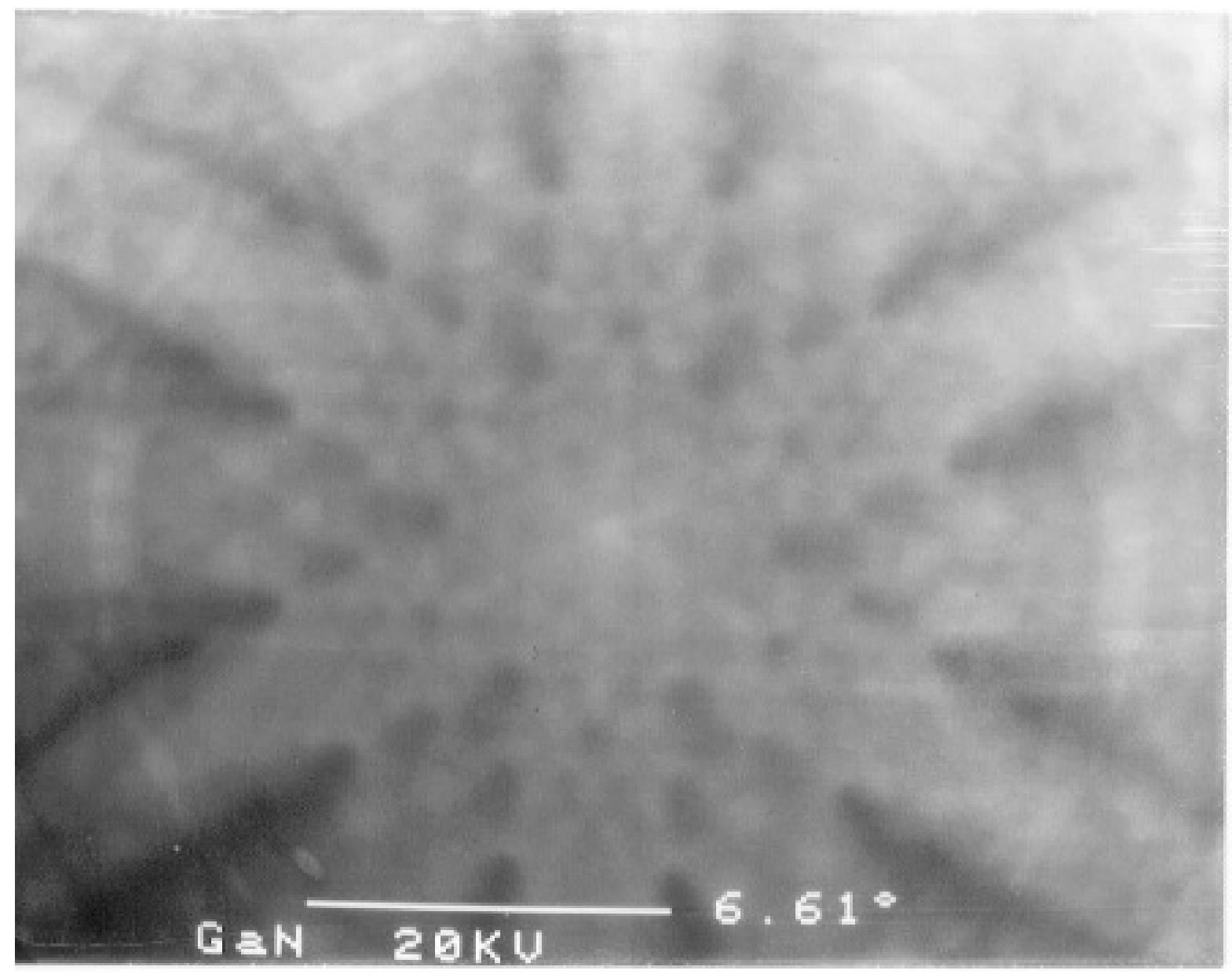

Figure 2b. Electron channeling pattern obtained from an area including both islands and background material.

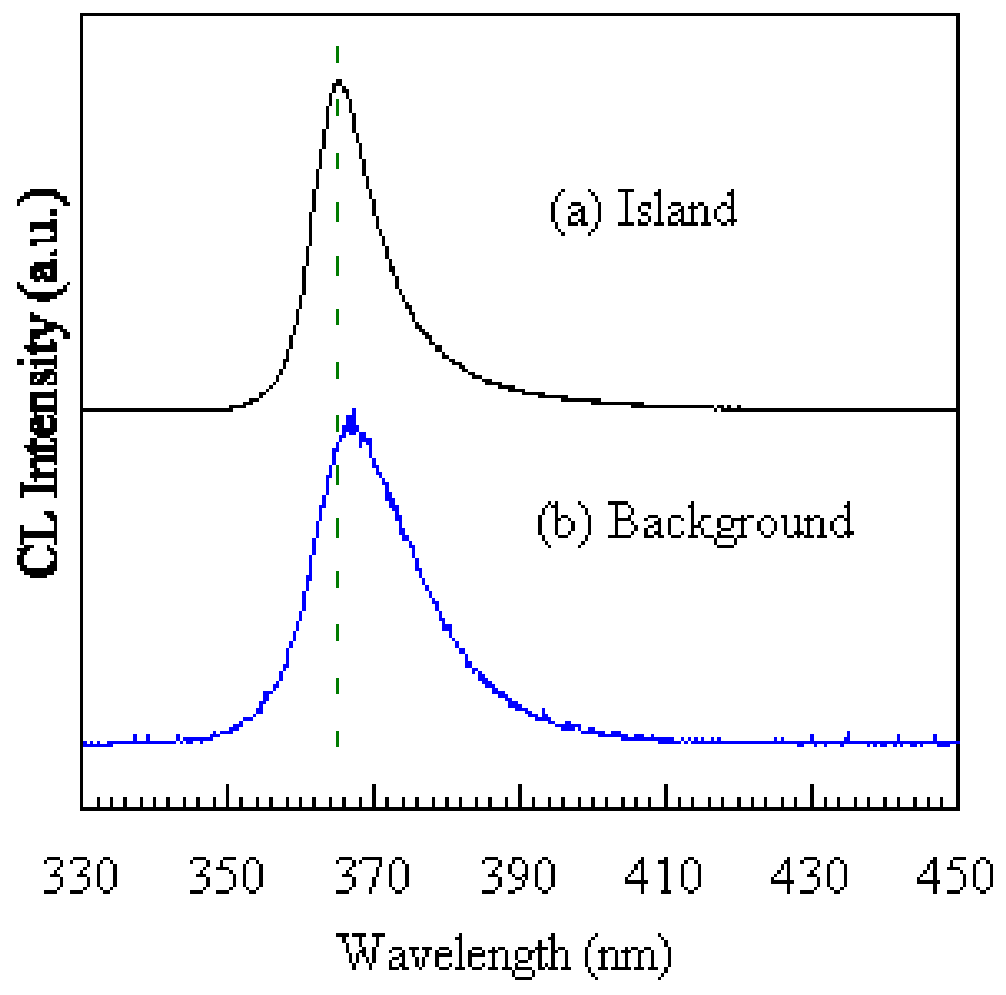

Figure 3. CL spectra at room temperature from (a) one of the islands and (b) its neighboring background material. 


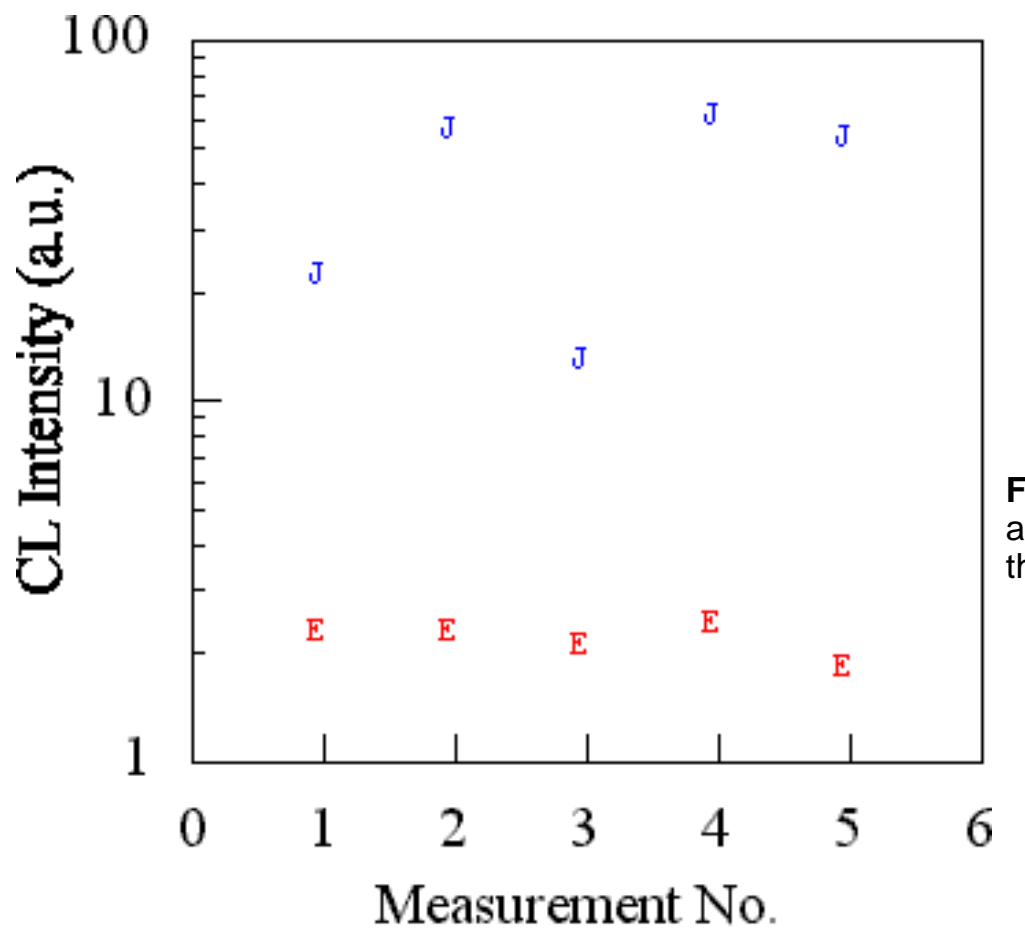

Figure 4. Comparison of luminescence efficiency at room temperature between five islands and their corresponding neighborhoods.
J Island
E Background 


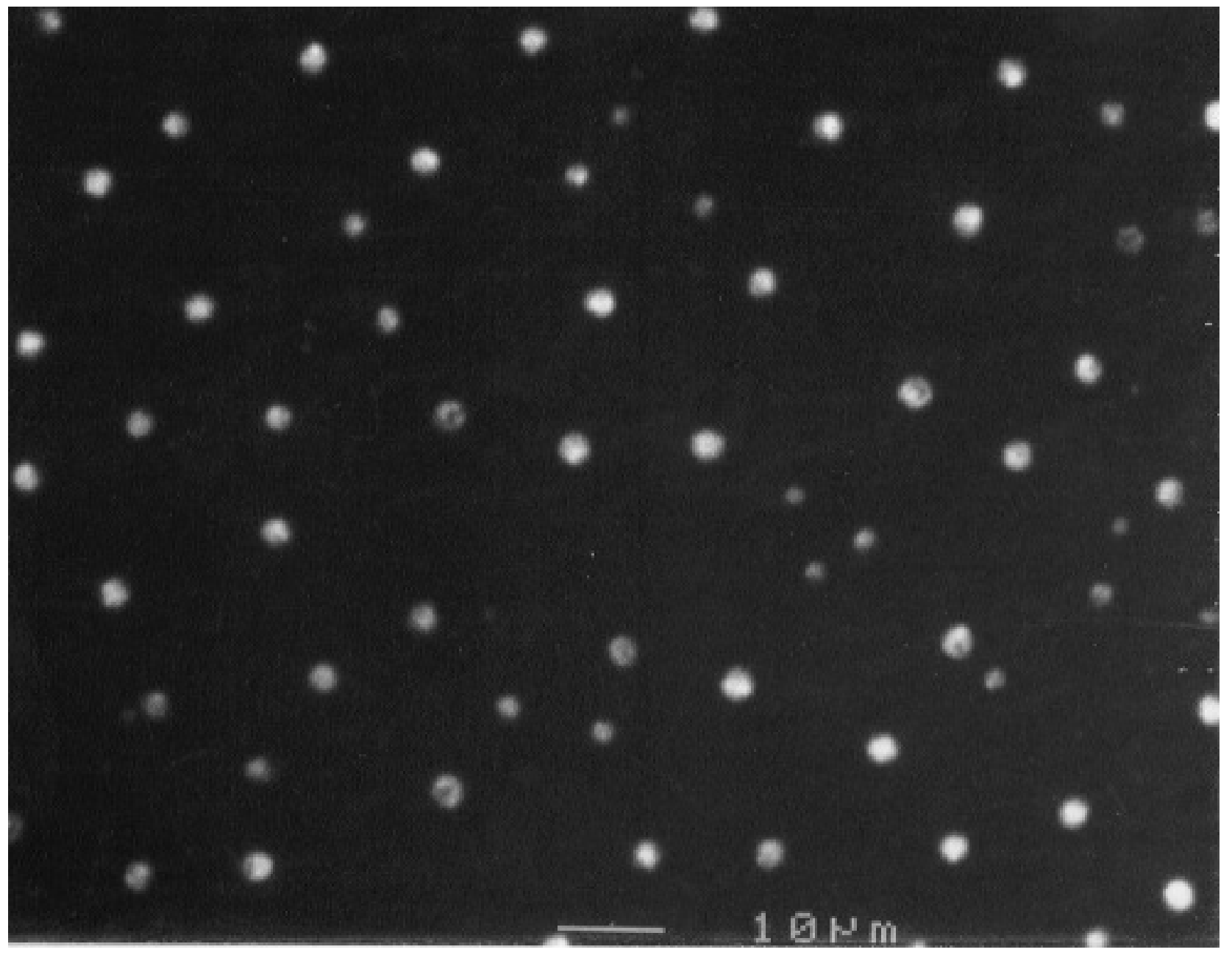

Figure 5. Room-temperature monochromatic $\mathrm{CL}$ image at $365 \mathrm{~nm}$ from the same area shown in the SEM image in Figure 1a. 


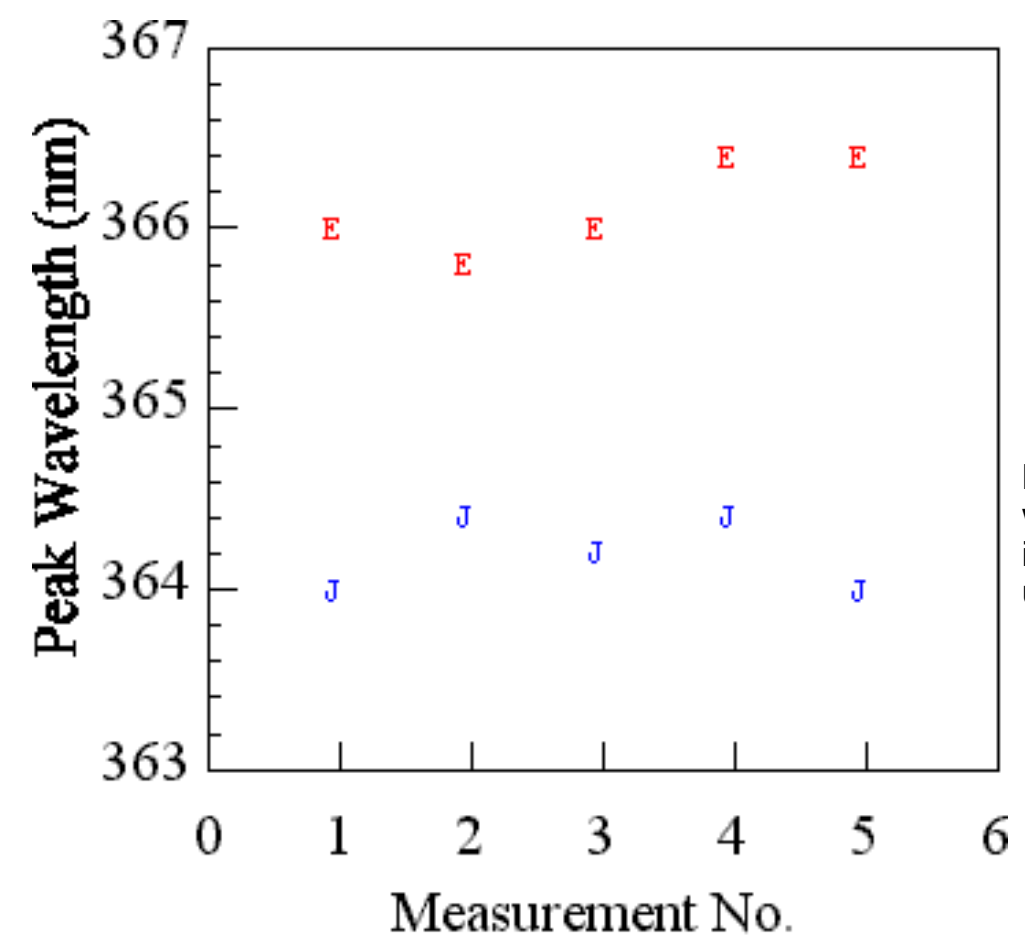

Figure 6. Comparison of peak luminescence wavelength at room temperature between five islands and their corresponding neighborhoods used in Figure 4.

J Island E Background

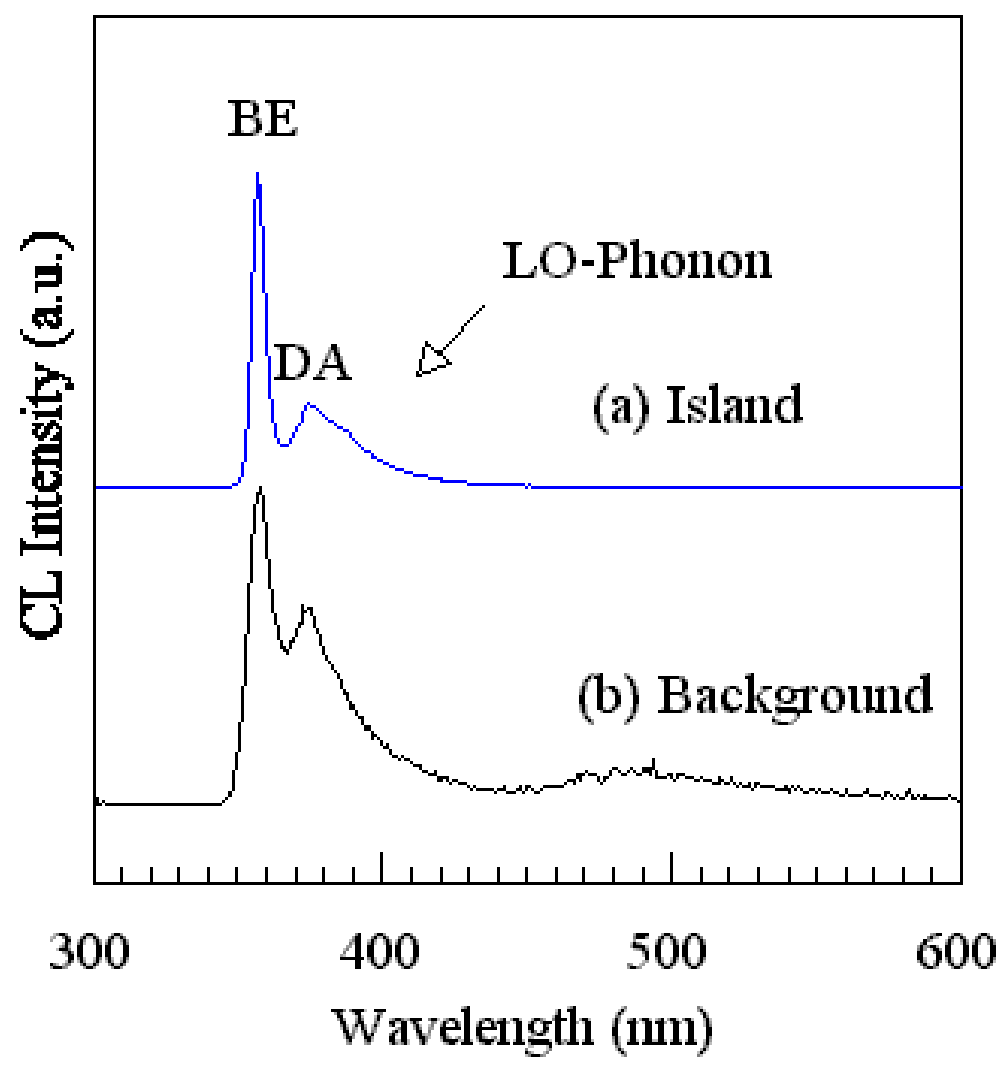

Figure 7. CL spectra at $8 \mathrm{~K}$ from (a) one of the islands and (b) its neighboring background material. 


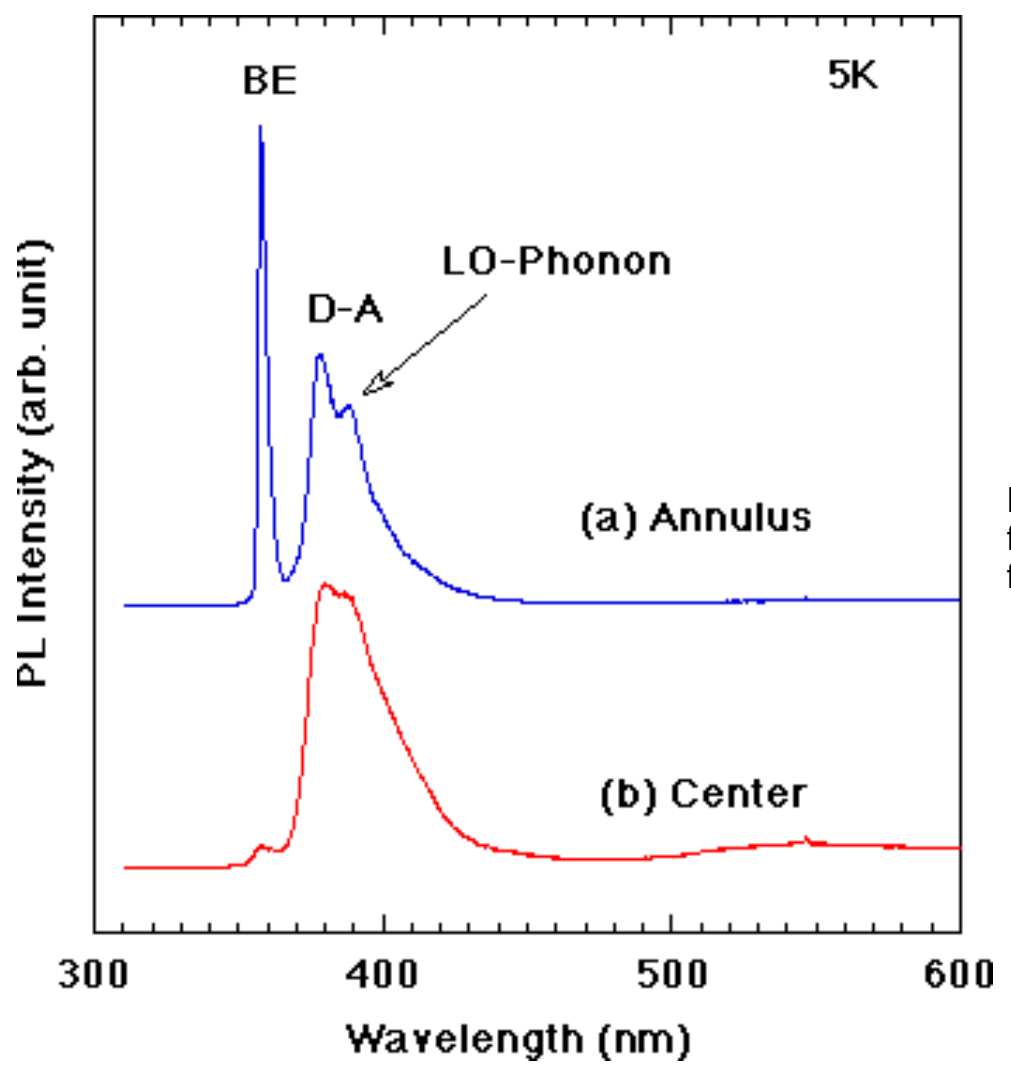

Figure 8. Photoluminescence spectra (a) at $5 \mathrm{~K}$ from the annular region with faceted islands (b) from the smooth, featureless central region.

(C) 1997 The Materials Research Society

\begin{tabular}{|l|l|l|l|l}
\hline $\mathrm{M}$ & $\mathrm{R}$ & $\mathrm{S}$ & Internet Journal of & Nitride Semiconductor Research \\
\hline
\end{tabular}

